\title{
Magnetic structure and electronic transport in permalloy
}

D. M. C. Nicholson, W. H. Butler, W. A. Shelton, Yang Wang,

X.-G. Zhang, and G. M. Stocks

Oak Ridge National Laboratory, Oak Ridge, TN 37831-6114

J. M. MacLaren

Dept. of Physics, Tulane University, New Orleans, LA 70118

\begin{abstract}
Permalloy $\left(\mathrm{Ni}_{.8} \mathrm{Fe}_{.2}\right)$ is an important alloy for magnetic devices. We present calculations of the electronic and magnetic structure of permalloy and of an interdiffused interface between permalloy and copper. We also present the results of calculations of the transport properties of permalloy. We find that the magnetic moments in permalloy are collinear, however, there may be non-collinear moments near an interface between permalloy and copper. We find that the calculated electrical conductivity is very sensitive to the details of the electronic and magnetic structure, however calculations which include relativistic effects yield a value for the conductivity that is in reasonable agreement with experiment.
\end{abstract}

PACS numbers: 73.61.-r, 75.70.Cn, 75.50.Rri,75.50.-i

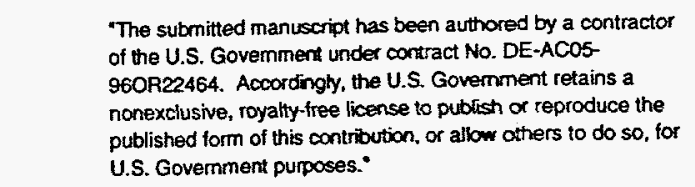

DISTRIBUTION OF THIS DOCUMENT IS UNLMITED

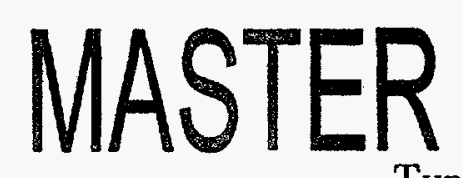




\section{DISCLAMMER}

Portions of this document may be illegible in electronic image products. Images are produced from the best available original document. 


\section{INTRODUCTION}

Permalloy $\left(\mathrm{Ni}_{.8} \mathrm{Fe}_{.2}\right)$ is an extremely important alloy for magnetic device applications because of its extremely high permeability, its extremely low magnetostriction, and its high anisotropic magnetoresistance. It has another interesting attribute that may be important for applications; it is an extremely good conductor for majority electrons and an extremely poor conductor for minority electrons.

This large asymmetry in conductivity results directly from its electronic and magnetic structure. In this paper we present calculations of the magnetic structure of the alloy including the effects of short-range order and local displacements. We also investigate the possibility that the magnetic moments in permalloy may be non-collinear. Our results indicate that this may occur at an interface with copper in which there has been interdiffusion.

We also present calculated results for the electrical conductivity of permalloy. We find that the calculated conductivity is extremely sensitive to the electronic and magnetic structure as well as to the lattice constant. By including scalar relativistic effects as well as spin-orbit coupling we find a value for the conductivity that is in reasonable agreement with experiment.

\section{MAGNETIC STRUCTURE OF PY AND CU-PY INTERFACES}

In this section we describe two models for permalloy and a model of permalloy-copper interfaces. Our first model of permalloy was designed to represent it as a random disordered alloy. The random alloy "sample" was prepared by placing 108 atoms on an fcc lattice. The sites were occupied by $\mathrm{Fe}$ with a probability of 0.2 and by $\mathrm{Ni}$ with probability 0.8 . This resulted in a sample with $88 \mathrm{Ni}$ and $20 \mathrm{Fe}$ atoms.

The second model of permalloy was designed to represent an alloy with short range order (SRO) arising both from non-random site occupation and from local displacements of the atoms due to their local environments. The second sample was constrained to have 
$88 \mathrm{Ni}$ and $20 \mathrm{Fe}$ atoms, so that the results of calculations using it could be compared directly to the results of calculations using the random sample. The site occupations and atomic displacements from the ideal lattice positions were obtained by using a Monte Carlo procedure which randomly interchanged and displaced atoms until agreement was obtained with experimental SRO parameters and displacements [1].

In order to determine the electronic and magnetic structure of these non-periodic systems we used the locally self-consistent multiple scattering technique [2]. For both samples, we assumed an fcc lattice parameter of $6.7 \mathrm{au}(3.545 \AA)$. The calculations were performed assuming collinear moments. The LSMS uses a local interaction zone (LIZ) for solving the Schrödinger equation. For these calculations this was taken to include four neighboring shells of atoms, a region large enough to converge the moments to $0.01 \mu_{B}$.

The distribution of moments as a function of unlike neighbors is shown Fig. 1. The spread in $\mathrm{Ni}$ and $\mathrm{Fe}$ moments due to their local environment is approximately $0.1 \mu_{B} . \mathrm{Ni}$ moments increase slightly on average with the number of neighboring $\mathrm{Fe}$ atoms. The $\mathrm{Fe}$ moment rises more rapidly with the number of $\mathrm{Ni}$ neighbors and there is less scatter from a linear dependence on Ni neighbors. The most notable difference between the two samples is that the average Fe moment has increased in the second sample. This is mainly caused by the larger number of $\mathrm{Ni}$ atoms around $\mathrm{Fe}$ in the $\mathrm{SRO}$ sample. There may also be an increase due to the displacement SRO which moves the Fe atoms away from one another. For a given number of $\mathrm{Ni}$ neighbors there is slightly more scatter in the moments on $\mathrm{Ni}$ and Fe in the SRO sample.

Because the current in permalloy is carried almost exclusively in the majority channel, it would be strongly affected by any deviations from collinearity in the $\mathrm{Ni}$ or $\mathrm{Fe}$ moments. In order to explore the possibility that environmental effects might induce non-collinear moments in permalloy, the moments in the converged collinear calculation for the first sample were redistributed at random over a solid angle extending to $\theta=9^{\circ}$. This new magnetic arrangement was iterated to self-consistency. Although convergence to a canted magnetic configuration was a possible outcome, the calculation converged to the collinear 
arrangement. This is an indication that non-collinearity of the moments is unlikely for bulk $\mathrm{Py}$ at $\mathrm{OK}$. It should be noted, however, that this calculation only traversed a very limited path through phase space and stable non-collinear arrangements may yet be found. Furthermore, the sample is small and does not include the unlikely Fe rich clusters associated with non-collinear moments in $\mathrm{Fe}_{.65} \mathrm{Ni}_{35}$ [3]. The concentrations of such clusters in Py would be too small to significantly affect the electrical conductivity.

We also investigated the possibility that there are non-collinear spins near a $\mathrm{Cu}-\mathrm{Py}$ interface. A model of a Cu-Py interface was constructed based on a previously described model for two atomic planes of $\mathrm{Ni}$ sandwiched between $\mathrm{Cu}$ layers [4]. In this model $\mathrm{Ni}$ and $\mathrm{Cu}$ layers were interdiffused. We calculated zero moment for this sample using the Coherent Potential Approximation. The Cu-Py model consists of eight [111] FCC planes each with 25 atoms. The sites were occupied with $\mathrm{Ni}, \mathrm{Cu}$, and $\mathrm{Fe}$ according to the concentration profiles used for the $\mathrm{NiCu}$. The concentration of $\mathrm{Fe}$ in a layer was taken to be one fourth the $\mathrm{Ni}$ concentration. The resulting sample contains $152 \mathrm{Cu}, 38 \mathrm{Ni}$, and $10 \mathrm{Fe}$ atoms.

A collinear LSMS calculation with a minimal LIZ, which included the first nearest neighbors yielded moments on $\mathrm{Ni}$ that varied widely depending on local environment. Ni moments varied from -.1 to $.3 \mu_{B}$. The fluctuations in the magnitude of the Fe moments, however, was small compared to their average value of $2.5 \mu_{B}$. The Ni moments increase with increasing number of $\mathrm{Fe}$ neighbors. The Fe moment shows no clear dependence on its neighbors. The calculated average moment per Py atom is $0.61 \mu_{B}$, whereas the total moment for sputtered films of similar geometry is observed to be zero at room temperature for such thin layers of Py [5].

In order to determine if the average moment could be reduced to zero in a non-collinear calculation we randomized the directions of the moments. A stable canted configuration was obtained with an average moment of $0.28 \mu_{B}$. The distribution of the magnitudes of the individual moments remained very similar to those in the collinear case. The directions of the Fe moments are distributed roughly between $\theta=0$ and $\theta=\pi / 2$ with respect to the overall magnetization. We could not discern a simple pattern relating the Fe canting angle 
to the local environment. As shown in Fig. 2, the Ni moment directions are spread over a wider range of $\theta$. A few are aligned approximately anti-parallel to the overall magnetization. Ths occurs only for sites with small moments. These sites had comparably small moments in the collinear calculation and are those sites with no $\mathrm{Fe}$ and few Ni neighbors. These results are consistent with comparisons of collinear and disordered local moment calculations that showed that the energy required to flip moments in $\mathrm{Py}$ is reduced by the addition of $\mathrm{Cu}$ [4]. The moments reorient themselves in response to subtle changes in their environments. It is likely that the magnetic structure will depend on the details of relaxation at the interface that are absent in these calculations. The energies of the canted and collinear configurations are closer than our numerical accuracy. We therefore postpone to a future publication a prediction of the ground state configuration. This calculation provides evidence that canted moments could arise at this type of interface. Such canted moments would be important for transport in layered magnetic materials.

\section{ELECTRONIC TRANSPORT}

In order to calculate the electrical resistivity of permalloy we used a simple semi-classical approximation [6] which we have shown agrees with the Kubo-Greenwood approach if the scattering is not too strong. Our approach is to calculate the electronic structure using the Coherent Potential Approximation (CPA). If the scattering is not too strong the CPA can be used to obtain energy bands with a non-zero width in energy which is proportional to the scattering rate (inverse lifetime). The energy bands can also be viewed for fixed energy as having a non-zero width in momentum which is proportional to the inverse of the mean free path. The integral of the mean free path over the Fermi surface can be shown to give the conductivity in the "lifetime" approximation. In these exploratory calculations we did not include the contributions to the conductivity from the "scattering-in" terms of the Boltzmann equation. We expect that the inclusion of such terms would raise the conductivity slightly. 
We have found the electrical resistivity of permalloy to be extremely sensitive to the details of the calculation. If the electronic structure is calculated using the non-relativistic Schrödinger equation there is a strong tendency for the $\mathrm{Ni}$ and Fe potentials to match almost perfectly in the majority channel. We calculated the resistivity of permalloy to be only $0.09 \mu \Omega \mathrm{cm}$ in this approximation [6], with essentially all of the current being carried by the majority electrons. This result is consistent with those of Mertig et al. who observed essentially zero resistivity for very dilute alloys of $\mathrm{Fe}$ in $\mathrm{Ni}$. We have found that scalar relativistic corrections to the the electronic structure can have a significant effect on the resistivity, raising it by a factor of two $(0.18 \mu \Omega \mathrm{cm}$ at the same lattice constant of $3.577 \AA)$. We also found the resistivity to be extremely sensitive to lattice constant, increasing from 0.18 to $0.30 \mu \Omega \mathrm{cm}$ for a $1 \%$ decrease in lattice constant. The main effect of the scalar relativistic corrections to the Schrödinger equation are to lower the $s$-band relative to the $d$-bands. This has the effect of bringing the Fermi energy closer to the $d$-bands which would be expected to increase the resistivity.

Not surprisingly, spin-orbit coupling has a large effect on the calculated resistivity because its inclusion couples the weak-scattering majority channel with the strong-scattering minority channel. We find that inclusion of spin-orbit coupling raises the resistivity from 0.30 to $2.22 \mu \Omega \mathrm{cm}$. This is still slightly less than the experimental value of approximately $4 \mu \Omega \mathrm{cm}$, but it is close to results that have been obtained recently using the Kubo-Greenwood formalism and including "vertex corrections" [7].

It is known that the single site Coherent Potential Approximation does not handle charge self-consistency perfectly $[8,9]$. It has been found that the potential on each site depends on its local environment, not just on the average concentrations of each species. The LSMS technique, however, makes it possible for us to investigate this effect. In order to study the effects of local environments on the electrical resistivity, we performed a CPA calculation in which each of the 108 different potentials obtained from the LSMS calculations was treated as a different species. The calculated resistivity without and with spin-orbit coupling was 0.41 and $2.61 \mu \Omega \mathrm{cm}$ respectively, which may be compared with the single site CPA values of 0.30 
and $2.22 \mu \Omega \mathrm{cm}$ at the same lattice constant $(3.5455 \AA)$. We also investigated the effects of short range order and local displacements on the resistivity. Our calculated resistivity using LSMS potentials generated with SRO and displacements was $0.31 \mu \Omega \mathrm{cm}$ without spin-orbit coupling and $2.38 \mu \Omega \mathrm{cm}$ including spin-orbit coupling.

We have not yet evaluated the effects on the resistivity of non-collinear spins and large variations in the magnetic moments induced by interdiffusion with copper at the interface, but we expect them to be large. This type of disorder will act to decrease the GMR in permalloy-Cu spin valves and multilayers.

\section{ACKNOWLEDGEMENTS}

Work at Oak Ridge was sponsored by the Division of Materials Sciences Office of Basic Energy Sciences and by the DOE Assistant Secretary of Defense Programs, Technology Management Group, Technology Transfer Initiative under contracts DEAC05-960R22464 with Lockheed Marietta Energy Research Corporation. Work at Tulane University was sponsored by the NSF Center for Photoinduced Processes. Most of the computations were performed at the ORNL Center for Computational Sciences. 


\section{REFERENCES}

[1] X. Jiang, G. E. Ice, C. J. Sparks, L. Roertson, and P. Zschack Phys. Rev. B 54, 3211 (1996).

[2] Yang Wang, G. M. Stocks, W. A. Shelton, D. M. C. Nicholson, Z. Szotek, and W. M. Temmerman, Phys. Rev. Lett. 75, 2867 (1995).

[3] Yang Wang, G. M. Stocks, W. A. Shelton, D. M. C. Nicholson, in this volume (1996).

[4] D. M. C. Nicholson, W. H. Butler, X.-G. Zhang, J. M. MacLaren, B. A. Gurney, and V. A. Seriosu, J. Appl. Phys. 76, 6805 (1994).

[5] V. S. Speriosu, J. P. Nozieres, B. A. Gurney,B. Dieny, T. C. Huang, and H. Lefakis, Phys. Rev. B 47, 11579 (1993).

[6] W. H. Butler, James M. MacLaren, and X.-G. Zhang, Mat. Res. Soc. Symp. Proc. 313 $59(1993)$

[7] John Banhart, A. Vernes, and H. Ebert, Sol. State. Comm. 98, 129 (1996).

[8] R. Magri, S.-H. Wei, and Alex Zunger, Phys. Rev. 4211388 (1990).

[9] D. D. Johnson and F. J. Pinski, Phys. Rev. 4811553 (1993). 


\section{FIGURES}

Fig.1 The spin magnetic moment as calculated with the LSMS for the Py sample with no $\mathrm{SRO}$ as a function of unlike nearest neighbors. The filled and unfilled circles represent $\mathrm{Ni}$ and $\mathrm{Fe}$ moments respectively.

Fig.2 Cotangents of the $\mathrm{Fe} l=2$ phase shifts as a function of unlike nearest neighbors in the Py sample with no SRO.

Fig. 3 The cosine of the canting angle relative to the direction of average magnetization as a function of the magnitude of the moment on the site (diamonds are $\mathrm{Ni} ;+$ are $\mathrm{Fe} ; \mathrm{Fe}$ moments are shifted by $2 \mu_{B}$ ) 


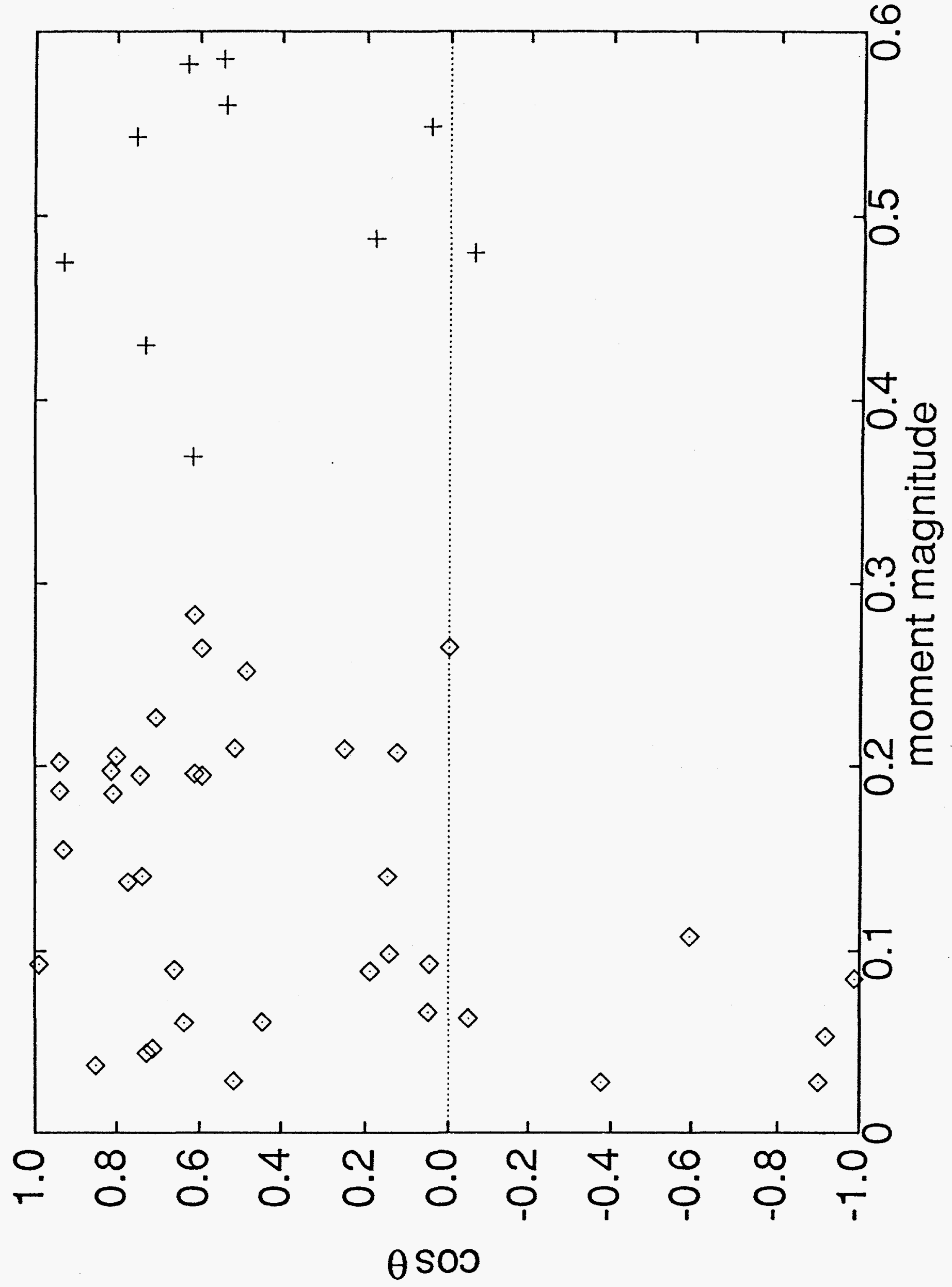


Spin magnetic moment $\mu$

B

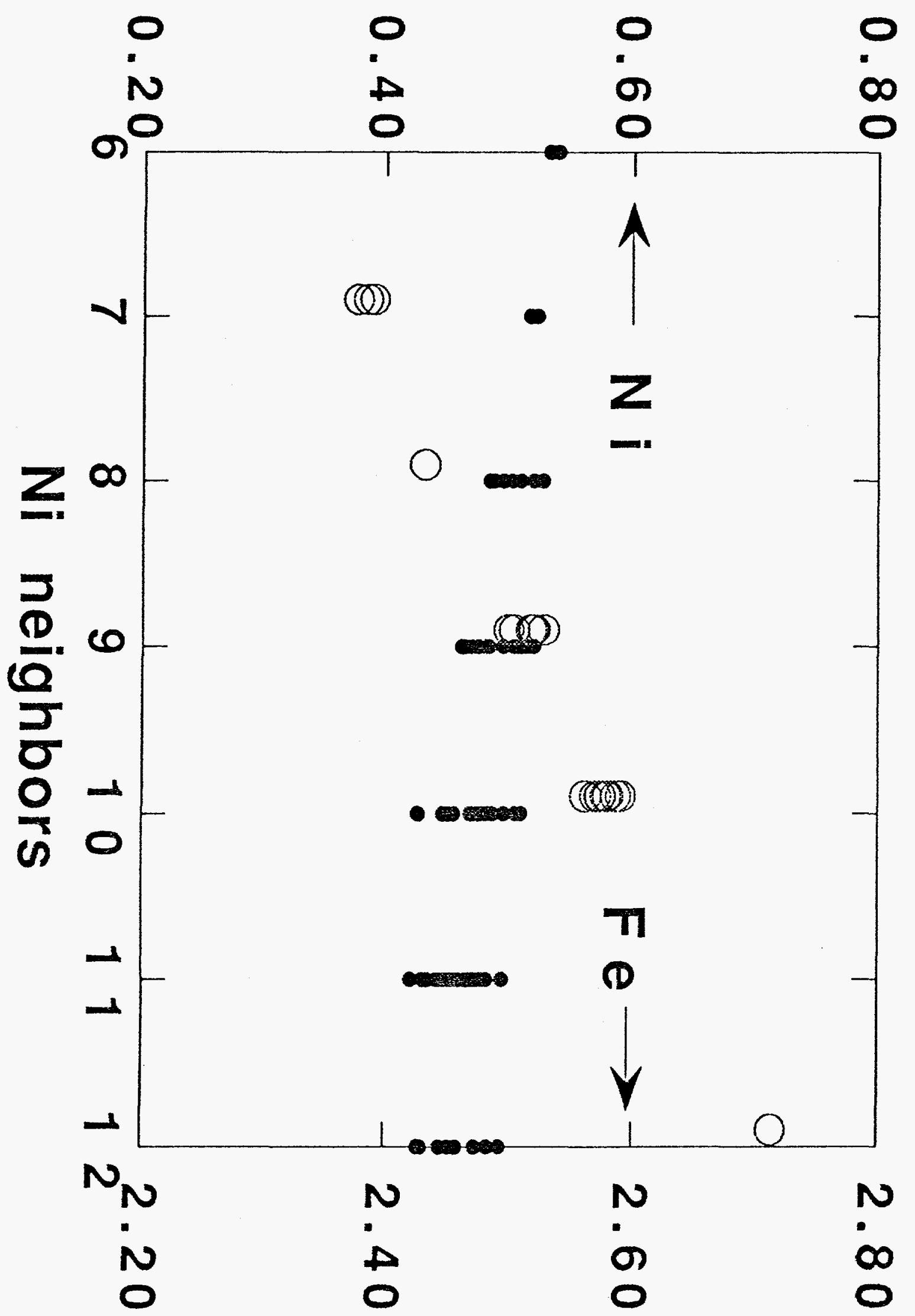




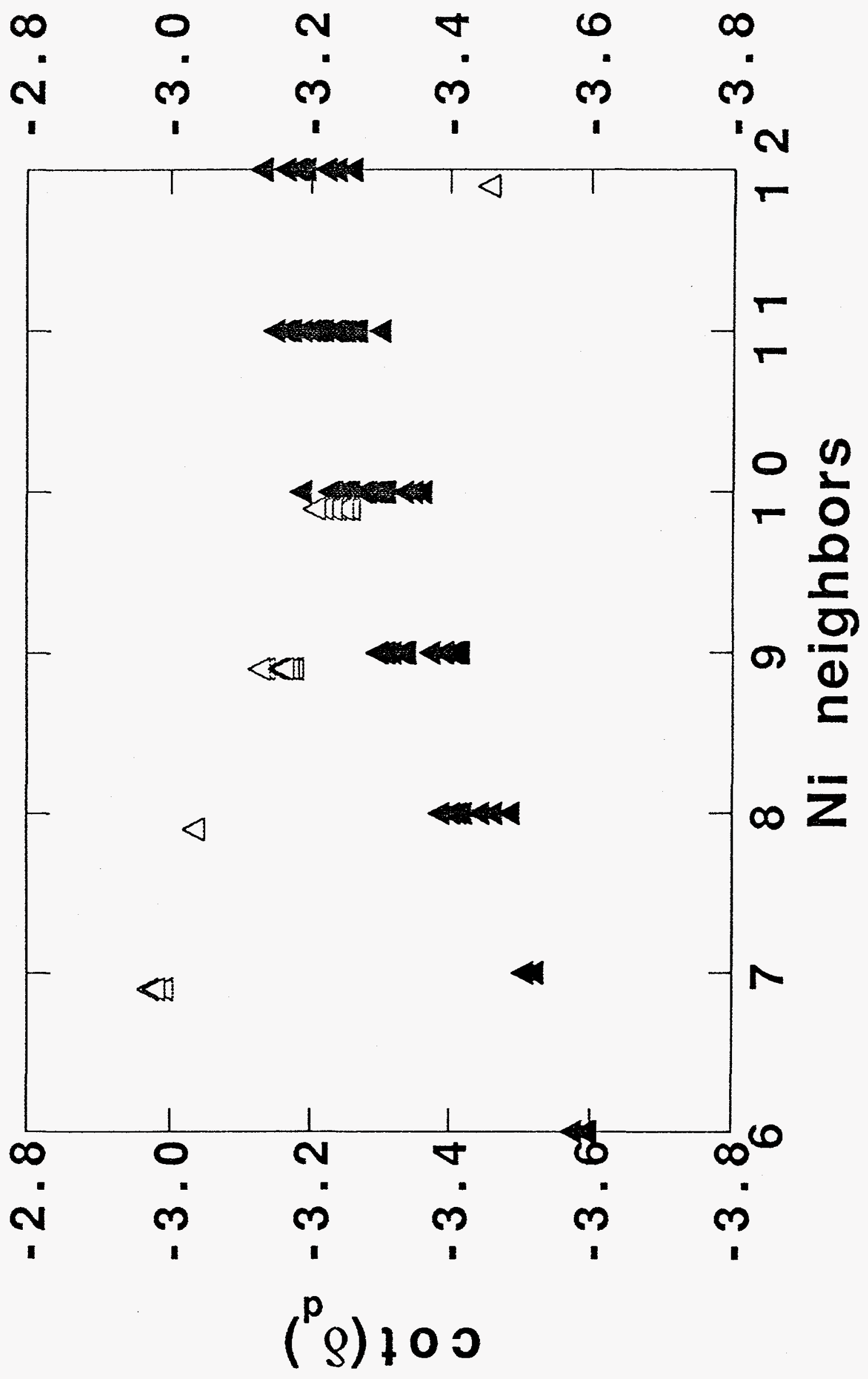




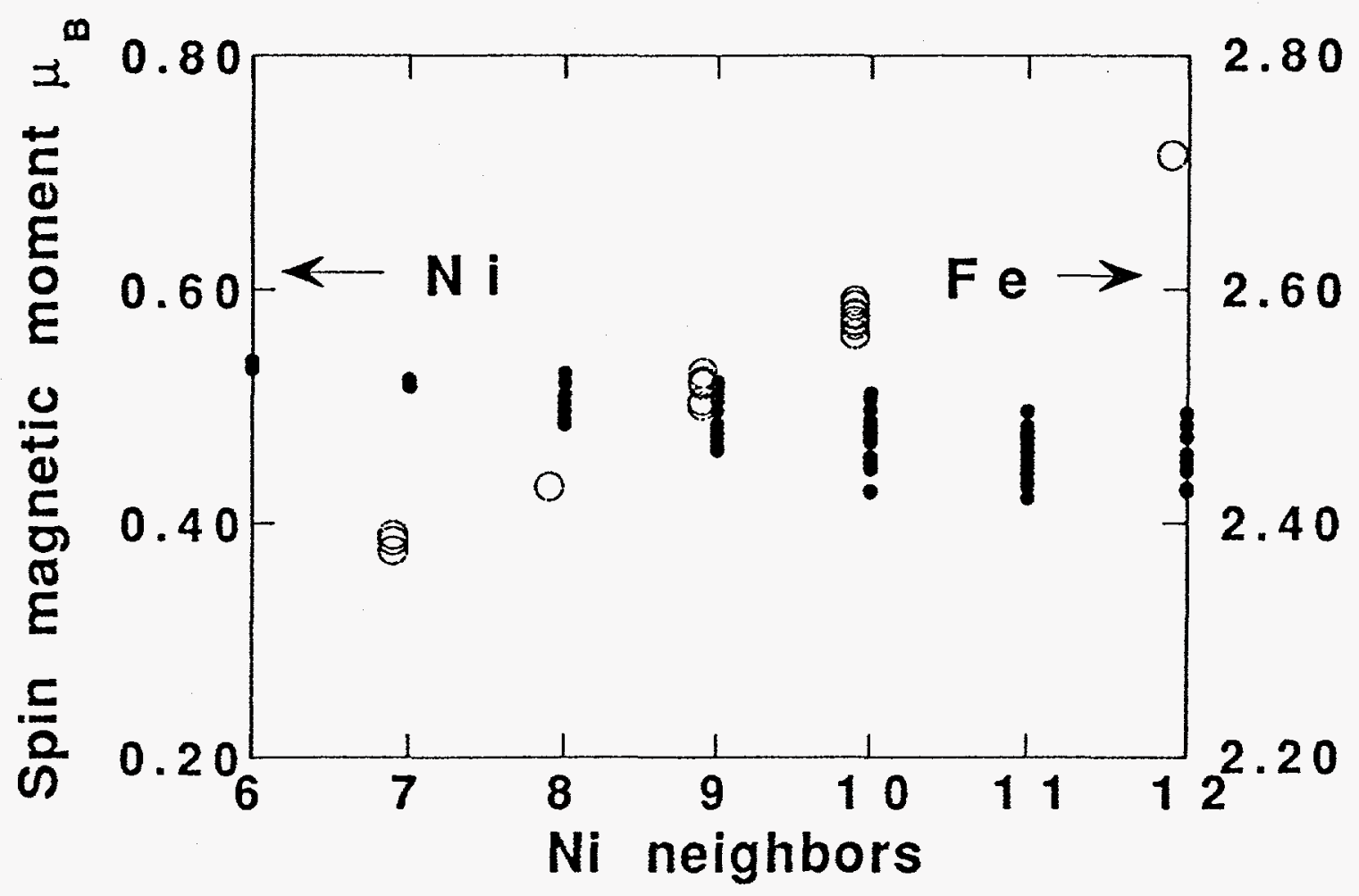




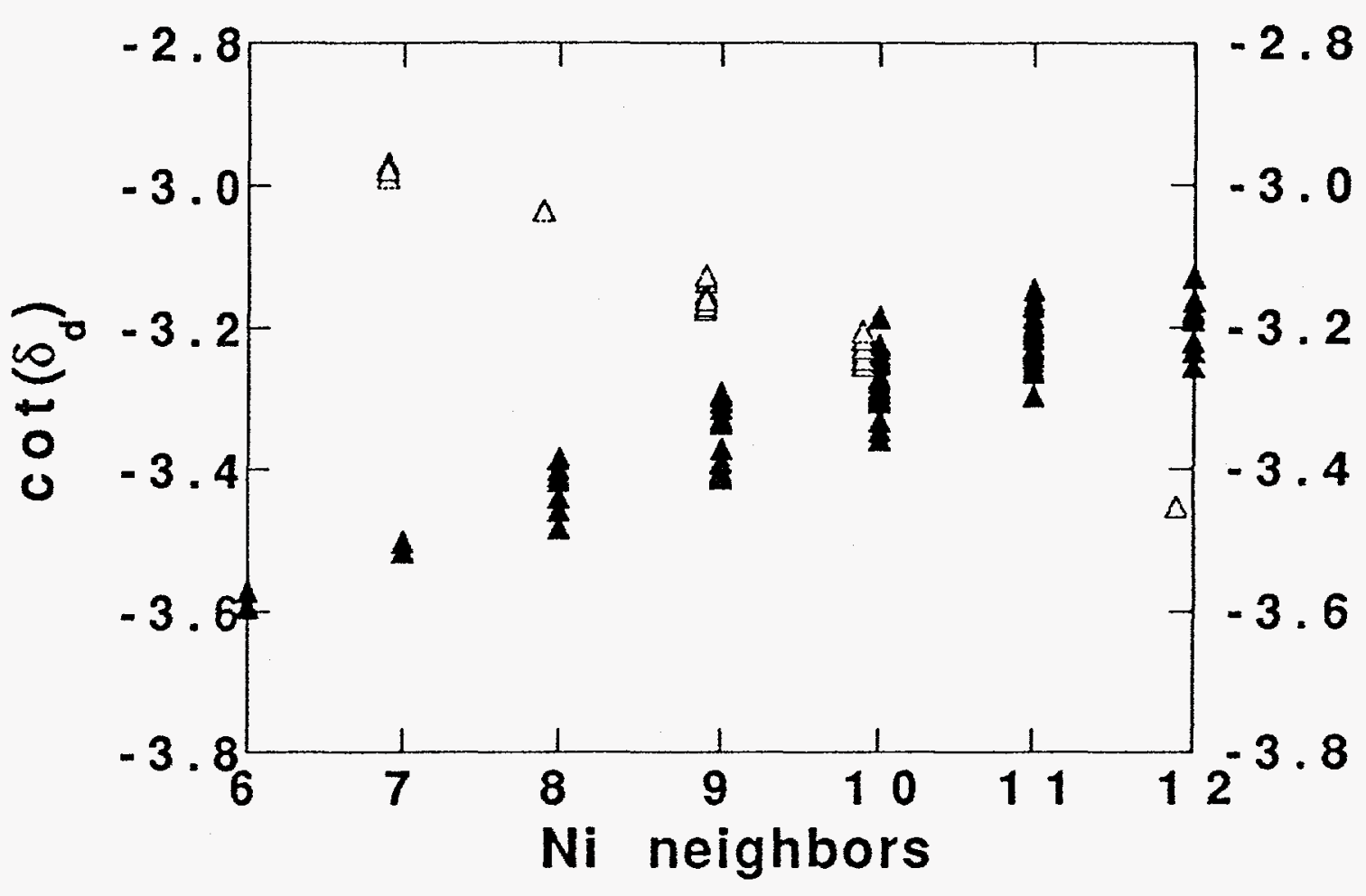

DISCLAIMER

This report was prepared as an account of work sponsored by an agency of the United States Government. Neither the United States Government nor any agency thereof, nor any of their employees, makes any warranty, express or implied, or assumes any legal liability or responsibility for the accuracy, completeness, or usefulness of any information, apparatus, product, or process disclosed, or represents that its use would not infringe privately owned rights. Reference herein to any specific commercial product, process, or service by trade name, trademark, manufacturer, or otherwise does not necessarily constitute or imply its endorsement, recommendation, or favoring by the United States Government or any agency thereof. The views and opinions of authors expressed herein do not necessarily state or reflect those of the United States Government or any agency thereof. 